
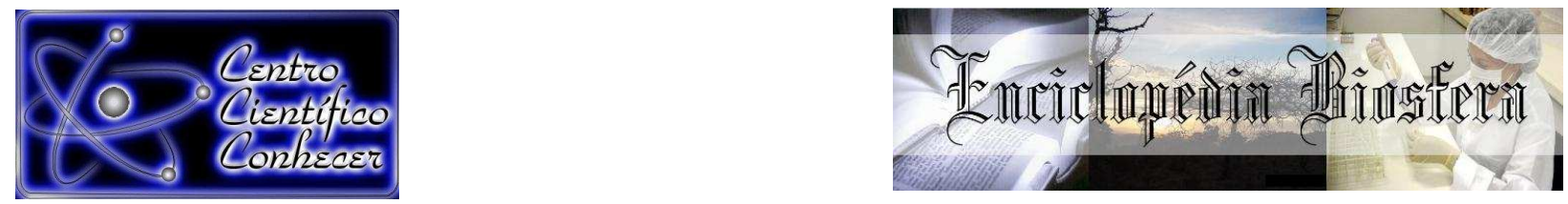

\title{
A INFLUÊNCIA DA GEOMETRIA DO CÂNION URBANO SOBRE O MICROCLIMA: UM ESTUDO PARA CIDADE DE CUIABÁ-MT
}

\author{
Fernanda Miguel Franco ${ }^{1}$; Marta Cristina de Jesus Albuquerque Nogueira ${ }^{2}$; Luiz \\ Orlando dos Santos ${ }^{3}$
}

1 Professora Doutora do Instituto Federal de Educação, Ciência e Tecnologia de Mato Grosso (fermifran@yahoo.com.br) Campus Cáceres, Cáceres-Brasil

2 Professora Titular do Departamento de Arquitetura e Urbanismo da Universidade Federal de Mato Grosso

3 Aluno do Instituto Federal de Educação, Ciência e Tecnologia de Mato Grosso Campus Cáceres

Recebido em: 08/09/2015 - Aprovado em: 14/11/2015 - Publicado em: 01/12/2015 DOI: http://dx.doi.org/10.18677/Enciclopedia_Biosfera_2015_058

\begin{abstract}
RESUMO
O crescimento urbano juntamente com o planejamento das cidades, tem dado pouca importância ao conforto ambiental urbano. Muitas vezes, as estratégias de elaboração de um projeto usado em países quentes secos, são oriundos de países de climas temperados e são, portanto, mal adaptados ao clima local. Segundo BOURBIA (2010), o planejamento urbano tem um imenso impacto sobre o microclima local, que, por sua vez, afeta o conforto e qualidade do espaço dentro de uma cidade. Como consequência do planejamento das cidades feito de forma errônea, as áreas urbanas, muitas vezes tornam-se desconfortáveis, resultando no agravo das condições microclimáticas, que acontece em decorrência de vários fatores, como o incremento urbano, a supressão da vegetação pré-existente, a poluição e canalização dos cursos d'agua, e a impermeabilização do solo. $O$ presente trabalho teve como objetivo avaliar e comparar a forma como a variação do microclima urbano pode afetar o ambiente construído, analisando as características dos cânions urbanos em três regiões na cidade de Cuiabá. Para tal, foram realizadas coletas de variáveis micrometeorológicos por meio de um transecto móvel em duas estações diferentes, uma quente-seca e outra quente-úmida. O clima de Cuiabá é do tipo AW de Koppen, isto é, Tropical semiúmido. O fator cânion urbano foi considerado representativo no estudo do desempenho microclimático, pois o mesmo pode ser responsável pela quantidade de radiação solar incidente, e, consequentemente, pelo aquecimento. A pesquisa apresenta fatores que acarretam mudanças no panorama climático urbano. Sendo assim, o estudo indica algumas questões inerentes ao processo de crescimento da capital mato-grossense, que necessitam ser revistos pelos gestores, a fim de uma melhor tomada de decisão com relação ao conforto ambiental da cidade.
\end{abstract}

PALAVRAS-CHAVE: Clima Urbano, Conforto Térmico, Traçado Urbano. 


\title{
THE INFLUENCE OF URBAN CANYON GEOMETRY ON MICROCLIMATE A STUDY FOR CUIABÁ-MT CITY
}

\begin{abstract}
The urban growth along with the planning of cities, has not given the real attention to urban environmental comfort. Many times the strategies of design a project used in countries with hot and dry climate, come from temperate countries and are not well adapted to the local climate. According to Bourbia (2010), urban planning has a huge impact on the local microclimate, which in turn affects the quality and comfort of space in the city. As a result of not planning right the urban areas often become uncomfortable, resulting in the injury of the microclimate conditions, which happens due to several factors, such as urban growth, the elimination of pre-existing vegetation, pollution and channeling of watercourses and soil sealing. This study aimed to evaluate and compare how the change in the urban microclimate may affect the built environment, analyzing the characteristics of the urban canyons into three regions in the city of Cuiabá. For this purpose, samples were taken from Micrometeorological variables by means of a transect in two different mobile stations, one hot and dry and the other hot and wet. The climate of Cuiabá is the AW type of Koppen, it means, Tropical monsoon climate. The urban canyon factor in the study was a presentation of microclimatic performance, as the same may be responsible for incident solar radiation, and heating. The research presents factors that cause changes in the urban climate landscape. Thus, the study indicates some issues inherent in the growth process of the capital of Mato Grosso, which need to be saw by managers in order to make the best decision about regarding the environmental comfort of the city.
\end{abstract}

KEYWORDS: Thermal Comfort, Trace Urban, Urban Climate.

\section{INTRODUÇÃO}

O clima urbano e o conforto térmico são processos que recebem pouca importância nos procedimentos de planejamento e design das cidades. Muitas vezes, os regulamentos de planejamento urbano usados em países quentes secos, a maioria dos que estão no mundo em desenvolvimento, são importados de climas temperados e são, portanto, mal adaptados ao clima local. Além disso, podem ser inflexíveis e, consequentemente, limitar a possibilidade de concepção urbana. Devido a essa incoerência de planejamento, as áreas urbanas, muitas vezes tornamse desconfortáveis (SHASHUA-BAR et al., 2010).

As modificações do clima no ambiente urbano apontam algumas características físicas que têm efeito sobre esses locais. Entre elas, a localização numa determinada região, a densidade da área construída, a orientação e largura das ruas, a altura relativa das edificações, os parques urbanos e áreas verdes (FRANCO, 2013).

Problemas de incidência solar em relação à orientação da rua, altura da edificação, largura da via e latitude são alguns apontamentos apresentados como relevantes para o estudo do fator cânion urbano. OKE (1988) investigou a maneira pela qual o clima urbano e geometria da rua são relacionados, e, também, sugeriu que investigações fossem realizadas em cidade de latitude médias. $O$ efeito da geometria urbana e orientação sobre o acesso solar e condições de sombreamento para diferentes condições de latitude tem sido estudado pelo pesquisador GIVONI (1998). Enquanto outros estudos examinaram a relação entre a densidade urbana e incidência solar, em um esforço para investigar as opções de design urbano. 
A cidade de Cuiabá resultou da expansão paulista, que buscavam índios e ouro na direção do noroeste, seguindo principalmente o rio Tietê e Paraguai. Em 1960, 238 anos após o inicio do povoamento, o município contava com cerca de 50 mil habitantes, em 1970, tinha mais de 100 mil habitantes. Já em 1980, o censo demográfico do IBGE contabilizou mais de 200 mil habitantes. Cuiabá chegou a 1991 com 400 mil habitantes (CUIABÁ, 2012). A localização da cidade estudada possui um clima com temperatura média anual de $27,9^{\circ} \mathrm{C}$, definido por dois períodos diferentes um quente-seco e outro quente-úmido.

Observa-se na cidade de Cuiabá que há ainda dentro do perímetro urbano áreas que não foram ocupadas, os chamados vazios urbanos. Mesmo assim, novos empreendimentos em regiões suburbanas estão sendo construídos, causando um grave problema de mobilidade, que é o deslocamento das pessoas diariamente para estes locais. Sendo assim, a organização das vias só é pensada de forma a escoar o tráfego de veículos e não atende os princípios relacionados ao conforto ambiental (FRANCO, 2013).

O estudo apresentado contribui para avaliar as características do layout e ambiente do cânion urbano em bairros que ainda não estão totalmente estruturados, além disso, investiga o efeito da geometria nas condições microclimáticas. Segundo ANDREOU (2014), as conclusões deste tipo de trabalho podem contribuir na formulação de diretrizes de design urbano.

O objetivo do estudo foi realizar um levantamento das variáveis microclimáticas (temperatura do ar e radiação solar) em três bairros na região próxima ao rio Coxipó (Boa Esperança, Santa Cruz, Jardim Universitário), avaliando e comparando a forma como essas afetam o ambiente construído, e qual a influência da geometria das vias (fator cânion urbano) sobre elas.

\section{Área de Estudo}

\section{MATERIAL E MÉTODOS}

Cuiabá possui uma área de $3.538,17 \mathrm{~km}^{2}$ correspondendo $254,57 \mathrm{~km}^{2}$ à macrozona urbana (Lei $\left.\mathrm{n} .{ }^{\circ} 4.719 / 04\right)$ e $3.283,60 \mathrm{~km}^{2}$ à área rural, conforme ilustrado na figura 01 (CUIABÁ, 2012). Com a finalidade de realizar o presente estudo, foram selecionados três bairros da Região Leste da cidade de Cuiabá, Mato Grosso, áreas que passaram por um processo de urbanização, que se apresentou mais intenso nos últimos 30 anos.

O processo evolutivo urbano de Cuiabá permitiu com que a cidade fosse se expandindo horizontalmente, sem nenhum limitador geográfico. Novos bairros iam surgindo, muitos, concebidos de maneira irregular, fruto de invasão. O adensamento e verticalização não foram uma opção para planejadores. Dessa forma, notam-se grandes vazios urbanos, assim como bairros com fragmentos de mata nativa ainda preservada (FRANCO 2013).

A escolha dos bairros deu-se devido aos seus aspectos morfológicos: bairros que estão em uma região valorizada da cidade, mas que ainda possuem considerável área de vegetação nativa, pela proximidade ao Rio Coxipó. 


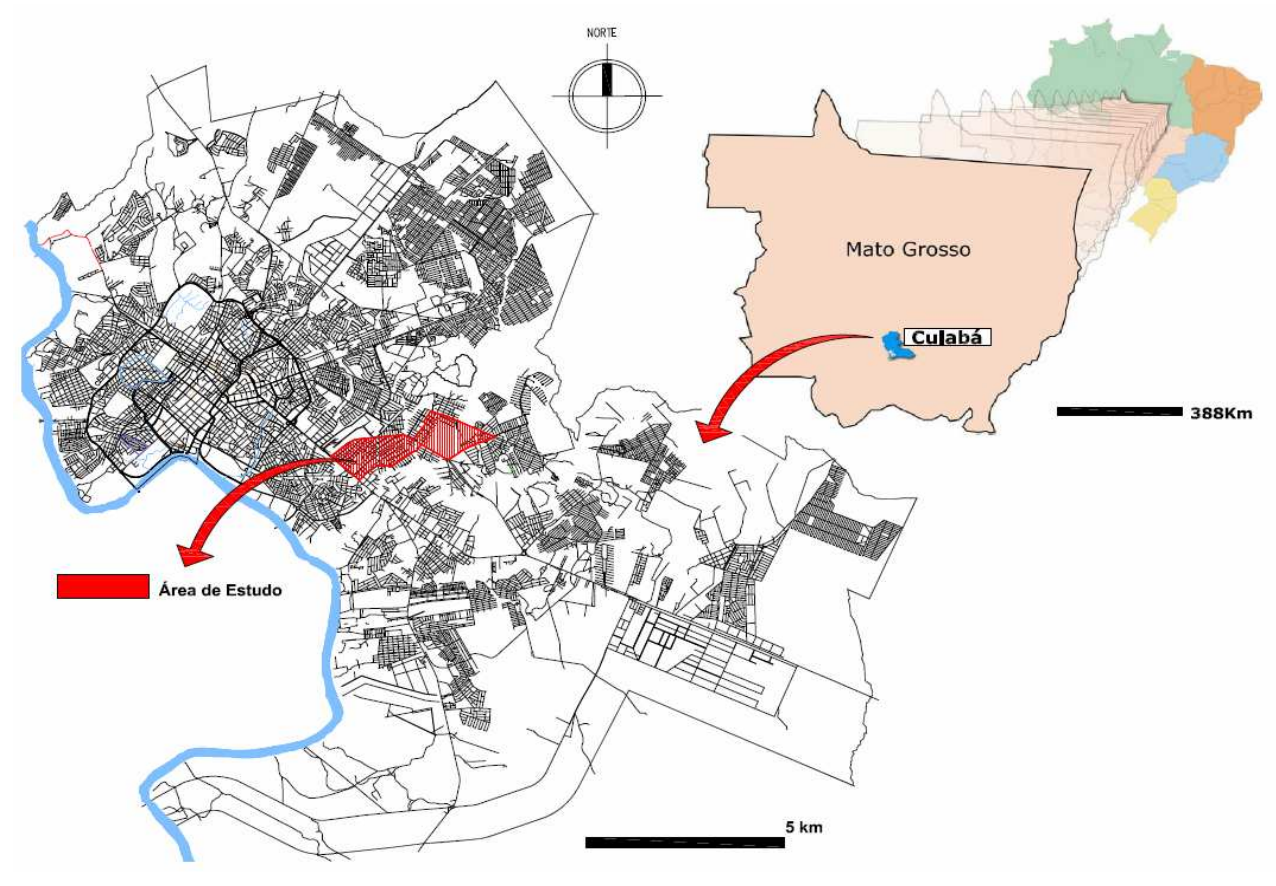

FIGURA 1 - Localização da região de estudo na cidade de Cuiabá-MT

Fonte: Adaptado, CUIABÁ (SMDU) 2012.

\section{Materiais utilizados}

A coleta de dados foi efetuada utilizando os seguintes equipamentos: Termômetro digital portátil datalogger Instrutherm modelo TH - 060, 2 canais de entrada (T1, T2,) escalas tipo K, capacidade de armazenamento: 15.000 dados, temperatura de operação: 0 a $40^{\circ} \mathrm{C}$. Sensor de par termoelétrico tipo K.

Para a realização do experimento o sensor tipo $\mathrm{K}$ foi instalado no interior de um abrigo, fabricado com dois tubos de PVC, o sistema também foi empregado por BARROS (2012). O sensor par foi abrigado no interior de um tubo de PVC com 2" de diâmetro por $50 \mathrm{~cm}$ de comprimento, e o conjunto foi acoplado no interior de um outro tubo de PVC, com 4" de diâmetro por $60 \mathrm{~cm}$ de comprimento. O sensor par foi fixado internamente ao tubo de 2" de diâmetro, de maneira a não tocar a parte metálica nas paredes do mesmo. Da mesma maneira, o tubo de 2" de diâmetro foi instalado no interior do tubo de 4" de diâmetro, possibilitando assim a ventilação entre eles (Figura 2A).

Para a aferição da variável radiação solar $\left(\mathrm{W} / \mathrm{m}^{2}\right)$ foi utilizado o medidor de energia solar da MS - 100 da Instrutherm (figura $2 \mathrm{~B}$ ), resolução de $1 \mathrm{~W} / \mathrm{m}^{2}, 1 \mathrm{Btu} /\left(\mathrm{ft}^{2} \mathrm{x}\right.$ h), escala de $2000 \mathrm{~W} / \mathrm{m}^{2}, 634 \mathrm{Btu} /\left(\mathrm{ft}^{2} \times \mathrm{h}\right)$, tempo de amostragem aprox. 0,4 segundo. As medições de todas as variáveis ocorreram consecutivamente nos pontos selecionados para o transecto móvel. 

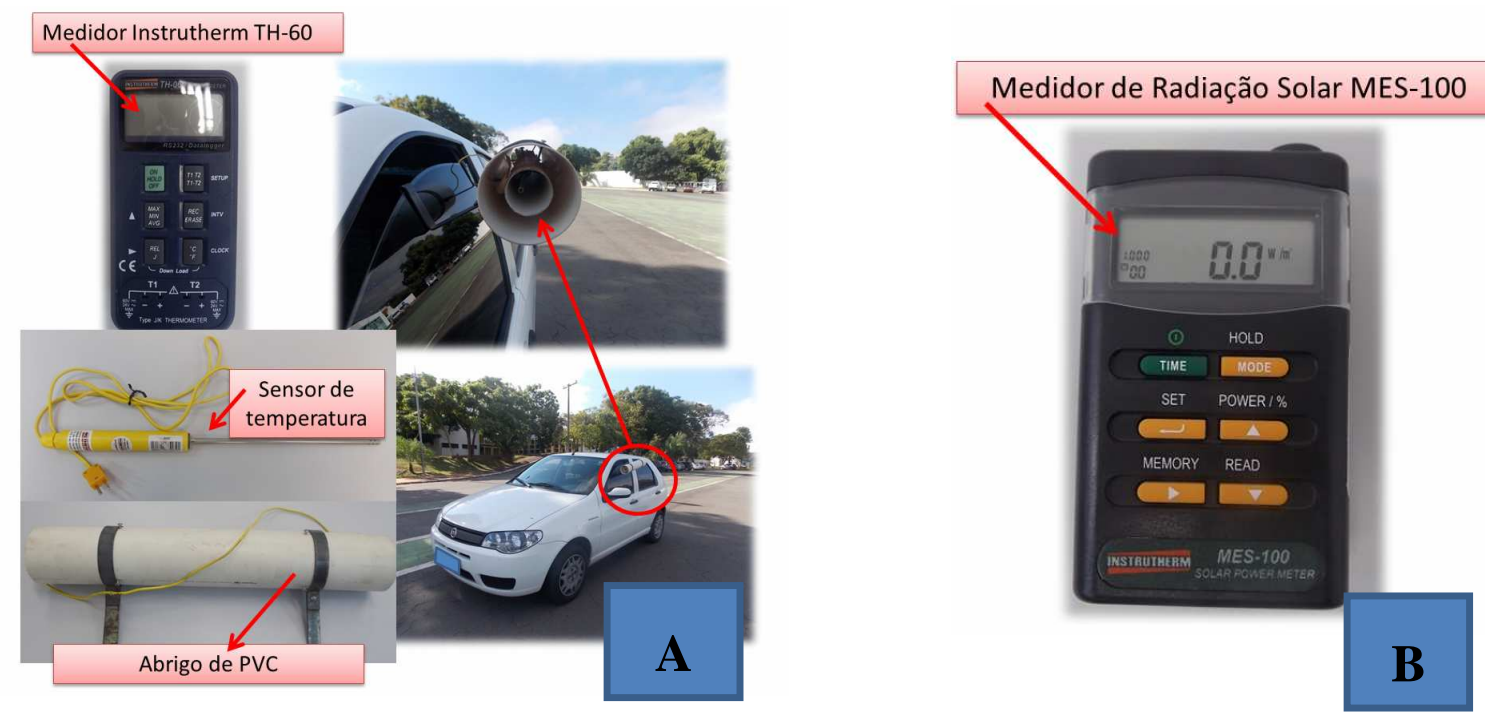

FIGURA 2 - (A) Instrumentos utilizados nas medições de temperatura do ar. (B) Medidor de Radiação Solar.

\section{Procedimento da coleta das variáveis ambientais}

Foram realizadas aferições de temperatura do ar e radiação solar por meio de um transecto móvel em distintas épocas do ano. A coleta das variáveis iniciou-se em setembro do ano de 2012, no período quente-seco, e finalizou em março de 2013, período quente-úmido. O experimento não aconteceu de maneira contínua, foram determinados dias em condições de tempo atmosférico ideal, com céu claro e ventos fracos nos períodos distintos, (Tabela 1 ).

O método de medições móveis e a instrumentação utilizada adaptaram-se com base nos trabalhos realizados por PEZZUTO (2007), em Campinas, SP, FRANCO (2010), e BARROS (2012) em Cuiabá, Mato Grosso.

TABELA 1- Período de coleta dos dados micrometeorológicos

\begin{tabular}{|c|r|r|r|}
\hline MÊS & DIA & NDIAS & PERÍODO \\
\hline SETEMBRO & $02 / 04 / 06 / 07 /$ & 10 & SECO \\
2012 & $09 / 11 / 13 / 19 / 23 / 30$ & 5 & ÚMIDO \\
\hline JANEIRO & $21 / 22 / 23 / 24 /$ & & \\
2013 & 25 & 5 & ÚMIDO \\
\hline MARÇO & $05 / 07 / 08 / 10 /$ & & \\
\hline
\end{tabular}


Determinado por um roteiro, as medições ocorriam cerca de 10 minutos de antecedência do horário proposto. De acordo com as condições do trânsito local, o veículo trafegava a uma velocidade média de $20 \mathrm{~km} / \mathrm{h}$, o itinerário era concluído em média com 50 minutos. Foi realizada uma parada de um minuto em cada um dos 23 pontos da área estudada, para que o equipamento pudesse se estabilizar.

O percurso do transecto móvel percorreu $10,3 \mathrm{~km}$ compreendendo as três regiões estudadas (figura 3 ).

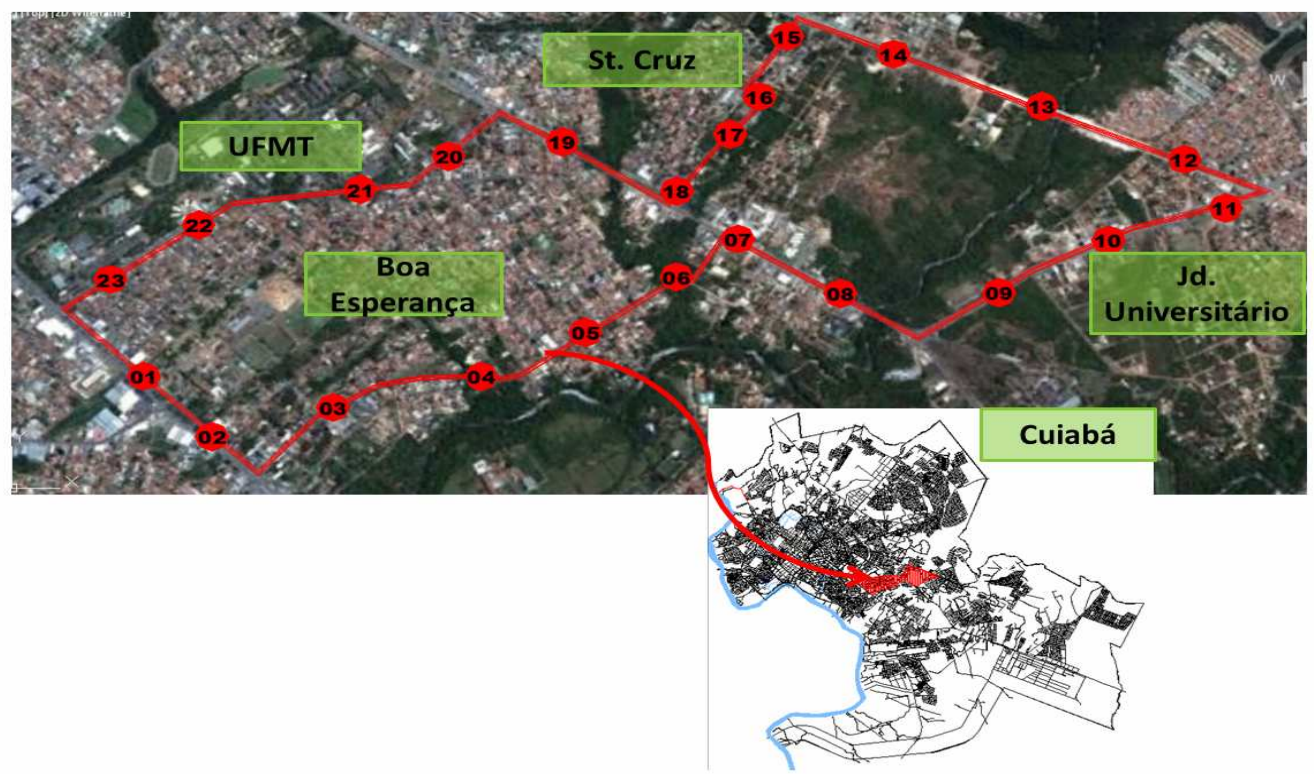
dados

FIGURA 3- Demarcação da região de estudo com os pontos da coleta de

\section{Caracterização do cânion urbano}

A medição das variáveis realizou-se de forma que possibilitasse fazer 0 cálculo do índice cânion urbano $(\mathrm{H} / \mathrm{W})$, que pode ser descrito como a relação entre a altura dos edifícios e a distância entre estes. Fator cânion urbano $(\mathrm{H} 1+\mathrm{H} 2 / \mathrm{W}), \mathrm{H} 1$ é a altura da edificação do lado direito, H2 é a altura da edificação do lado esquerdo e W a largura da rua.

Realizou-se o levantamento das alturas das edificações que se encontram do lado direito e esquerdo da via em que se posicionava cada ponto, assim como a largura da via. Para as medidas, utilizou-se uma fita métrica de 50 metros. Com essas informações foi possível fazer o cálculo do índice cânion urbano.

\section{RESULTADOS E DISCUSSÃO}

A influência do cânion urbano é significativa para formação da ilha de calor. Vários são os fatores que influenciam o microclima do cânion urbano, entre estes: o calor antropogênico produzido pela circulação de pessoas, pelo tráfego de veículo, pelo aquecimento das casas e prédios próximos. Quando se trata de um clima quente, como encontrado na cidade em estudo, há uma diminuição na perda de ondas longas (infravermelho), causada pela redução do fator visão do céu, pela geometria dos prédios. Há uma grande capacidade de estocar calor, bem como de reemiti-lo, causado pelas propriedades térmicas dos materiais de construção. Como consequência, tem-se um aumento do fluxo de calor sensível causado pela redução da evaporação, que, por sua vez, é causado pela ausência da vegetação e da ENCICLOPÉDIA BIOSFERA, Centro Científico Conhecer - Goiânia, v.11 n.22; p. 34662015 
superfície permeável, convergência do calor sensível, causada pela redução da velocidade do vento na camada intraurbana.

TABELA 2- Características do Canyon Urbano na cidade de Cuiabá

\begin{tabular}{|c|c|c|c|c|c|c|}
\hline \multicolumn{7}{|c|}{ CANYON URBANO } \\
\hline PONTOS & ALTITUDE & DIREÇÃO & $\begin{array}{c}\text { Altura } \\
\text { Edifício } \\
\text { Esq. }\end{array}$ & $\begin{array}{c}\text { Altura } \\
\text { Edifício } \\
\text { Dir. }\end{array}$ & $\begin{array}{c}\text { Largura da } \\
\text { Rua }\end{array}$ & H/W Fator \\
\hline P1 & 164 & NO-SE & 5,8 & 10 & 48,11 & 0,22 \\
\hline P2 & 178 & NO-SE & 10 & 7 & 60,02 & 0,22 \\
\hline P3 & 177 & SO-NE & 12 & 7 & 17,3 & 0,89 \\
\hline P4 & 159 & O-L & 10,5 & 12 & 6 & 2,75 \\
\hline P5 & 166 & OSO-ENE & 3 & 5,5 & 14,65 & 0,39 \\
\hline P6 & 163 & NO-SE & 12 & 15 & 6,6 & 2,95 \\
\hline P7 & 174 & NO-SE & 5 & 2,5 & 59,17 & 0,11 \\
\hline P8 & 169 & NO-SE & 10 & 5 & 28 & 0,45 \\
\hline P9 & 172 & SO-NE & 9,5 & 9 & 10,2 & 1,37 \\
\hline P10 & 177 & OSO-ENE & 22,4 & 3,5 & 32,3 & 0,75 \\
\hline P11 & 185 & OSO-ENE & 11,2 & 4 & 36,21 & 0,36 \\
\hline P12 & 178 & ESE-ONO & 0 & 3 & 39,15 & 0,04 \\
\hline P13 & 166 & ESE-ONO & 12 & 15 & 35,76 & 0,54 \\
\hline P14 & 186 & ESE-ONO & & 5 & 48,85 & 0,05 \\
\hline P15 & 188 & NNE-SSO & 1,7 & 3 & 14,22 & 0,22 \\
\hline & & ONO- & & & & \\
\hline P16 & 189 & ESSE & 2,3 & 7,5 & 11,75 & 0,51 \\
\hline P17 & 182 & NE-SO & 12 & 8 & 11,17 & 1,43 \\
\hline P18 & 170 & NNE-SSO & $*$ & $*$ & 8,75 & \\
\hline P19 & 171 & ESE-ONO & 3 & 6 & 40,05 & 0,15 \\
\hline P20 & 183 & NE-SO & 4,5 & 9 & 14,5 & 0,62 \\
\hline P21 & 184 & L-O & 10 & 9,5 & 25,2 & 0,58 \\
\hline P22 & 177 & ENE-OSO & 6 & 4 & 33,3 & 0,24 \\
\hline P23 & 177 & NE-SO & 3 & 9 & 56 & 0,13 \\
\hline & Noss & & & & \\
\hline
\end{tabular}

* Não foi possível fazer o cálculo do fator canyon urbano

Como a sombra pode bloquear a radiação solar incidente, alguns estudos têm discutido o efeito do sombreamento em ambientes internos e externos. A orientação da rua e a relação altura/largura $(\mathrm{H} / \mathrm{W})$ foram medidas para avaliar os níveis de 
sombreamento em estudos realizados por JOHANSSON (2006) e EMMANUEL et al. (2007). Ao longo do trajeto do transecto foram determinados 23 pontos onde se obteve também os valores das condicionantes físicas do cânion urbano (tabela 2).

A Figura 4 ilustra o comportamento da temperatura do ar e a relação deste com o fator cânion urbano $(\mathrm{H} / \mathrm{W})$. Nota-se que em uma mesma via os pontos 4, 5 e 6 , onde 4 e 6 tem um fator cânion mais elevado, há uma queda de aproximadamente $0,5^{\circ} \mathrm{C}$ em relação ao ponto 5 , sem contar que os pont os distam $100 \mathrm{~m}$ um do outro, um ganho bem significativo em se tratando de uma distância tão pequena.

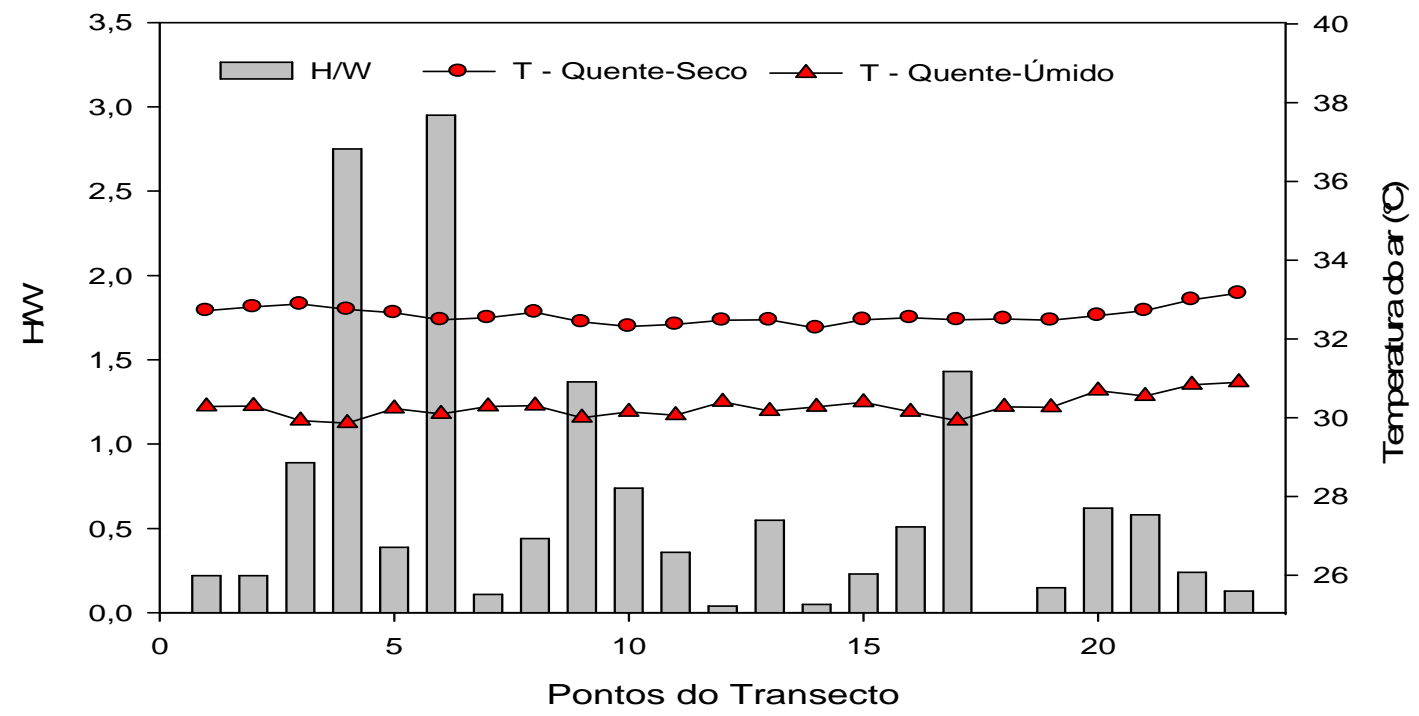

FIGURA 4 - Comparação entre a temperatura do ar média (período seco e úmido) e a relação $\mathrm{H} / \mathrm{W}$

O ponto 17, com a menor temperatura do ar no período quente-úmido $\left(29,9^{\circ} \mathrm{C}\right)$, é o $3^{\circ} \mathrm{com}$ o maior fator $(\mathrm{H} / \mathrm{W}=1,43)$, enqu anto o ponto $23 \mathrm{com}$ a maior temperatura do ar no período quente-úmido $\left(30,9^{\circ} \mathrm{C}\right)$, tem um fator $(\mathrm{H} / \mathrm{W}=0,13), 11$ vezes menor que o do ponto 17 (figura 04).

Outro fator importante que deve ser considerado é a direção em relação aos pontos cardeais que esse cânion está direcionado, no caso do ponto 17 encontra-se na direção NE-SO, o que significa que em determinadas horas do dia as construções e a vegetação vão sombrear a via. Os pontos 4 (O-L) e 5 (OSO-ENE) de elevado fator $(\mathrm{H} / \mathrm{W})$ estão em uma direção que não privilegia tanto o sombreamento, o ponto 4 ainda encontra-se em situação bem desfavorável com relação a isso, pois o sol incide sobre esse ponto ao longo de todo dia. No entanto, no ponto 4 há a influência da vegetação que está dos dois lados da rua fazendo assim um túnel verde.

Em um estudo realizado por KRÜGER et al. (2010), eles afirmam que em ruas $\mathrm{N}$-S com elevada proporção (H/W) pode garantir sombreamento mútuo das fachadas dos edifícios, resultando em reduções da necessidade de refrigeração. Todavia, as ruas largas, no eixo L-O têm elevado ganho de energia térmica ao longo do dia. $O$ fator abertura do cânion aliado à direção da rua pode garantir proteção tanto ao pedestre como as construções. 


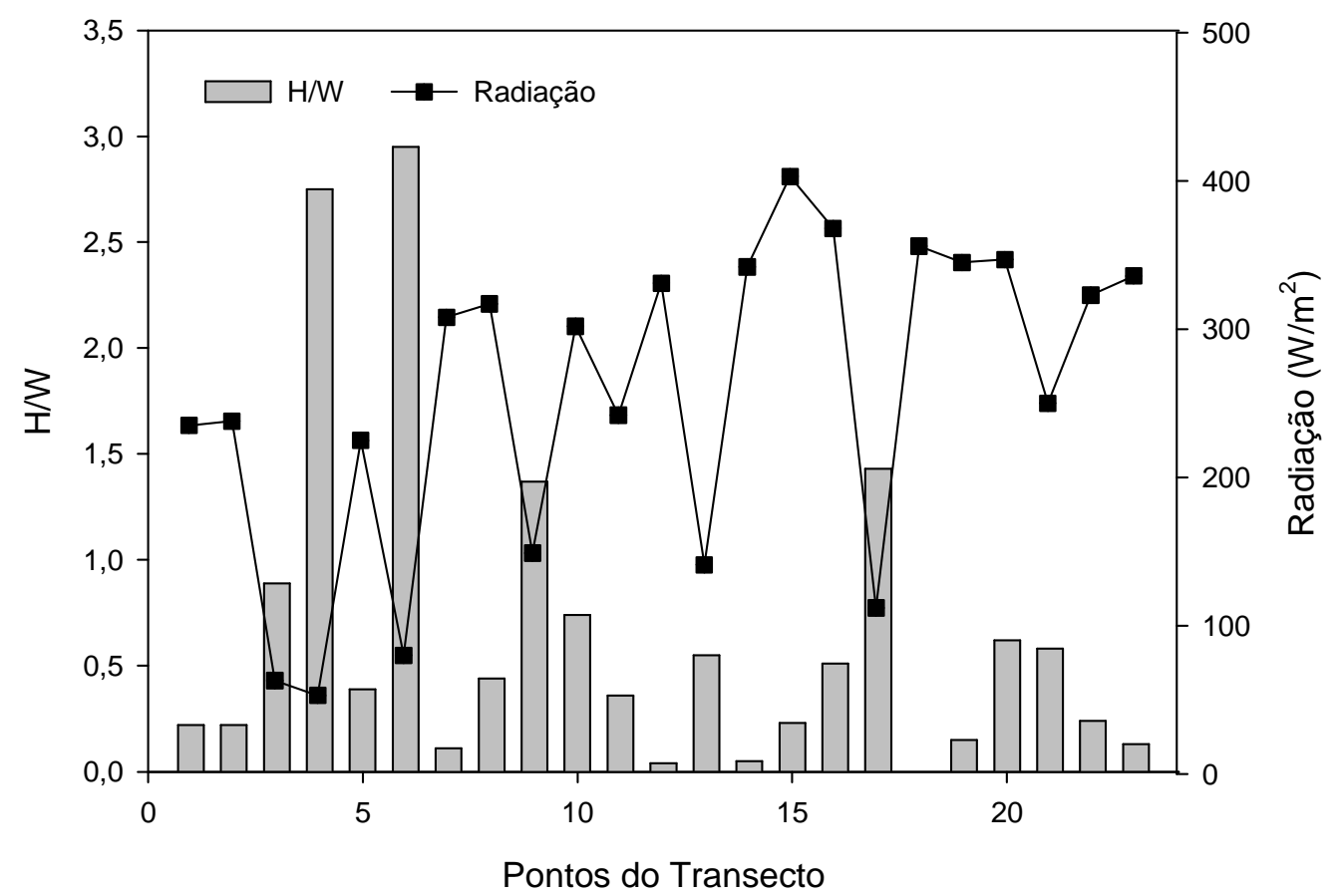

FIGURA 5 - Comparação entre Radiação e H/W

A radiação é outra variável intimamente ligada com o fator de abertura do cânion, notada nos pontos $3\left(63 \mathrm{~W} / \mathrm{m}^{2}\right.$ e 0,89$), 4\left(53 \mathrm{~W} / \mathrm{m}^{2}\right.$ e 2,75$), 6\left(80 \mathrm{~W} / \mathrm{m}^{2}\right.$ e 2,95$)$, $9\left(149 \mathrm{~W} / \mathrm{m}^{2}\right.$ e 1,37), $17\left(112 \mathrm{~W} / \mathrm{m}^{2}\right.$ e 1,43). Onde estão localizados os maiores fatores $(\mathrm{W} / \mathrm{H})$, as medidas de radiação solar também são menores (figura 5). A maior média de radiação solar foi aferida no ponto $15 \mathrm{com} 405 \mathrm{~W} / \mathrm{m}^{2}$ e fator $(\mathrm{H} / \mathrm{W})$ de 0,23 e a menor radiação solar no ponto $4 \mathrm{com} 53 \mathrm{~W} / \mathrm{m}^{2}$ e fator $(\mathrm{H} / \mathrm{W})$ de 2,75 . O fator de abertura do cânion juntamente com a direção pode determinar as horas de insolação que aquele local poderá ter.

No estudo, fica evidente a diferença de radiação solar entre o ponto 4 e 0 ponto 15 que é de $352 \mathrm{~W} / \mathrm{m}^{2}$, aproximadamente sete vezes maior. Na relação $\mathrm{H} / \mathrm{W}$ a diferença é de 2,52, aproximadamente 11 vezes maior (figura 6). PEARLMUTTER et al. (2007) afirmam que a abertura do cânion e sua direção têm efeito direto sobre a radiação solar e consequentemente no conforto exterior, e sugerem que cânion $(H / W)$ na faixa de 1,0-2,0 melhora significativamente o ambiente térmico urbano.

STROMANN-ANDERSEN \& SATTRUP (2011) calcularam que a geometria do cânion tem um impacto no consumo total de energia de até 30\% para edifícios comerciais e $19 \%$ para edifícios residenciais, somas consideráveis na busca por uma cidade mais sustentável.
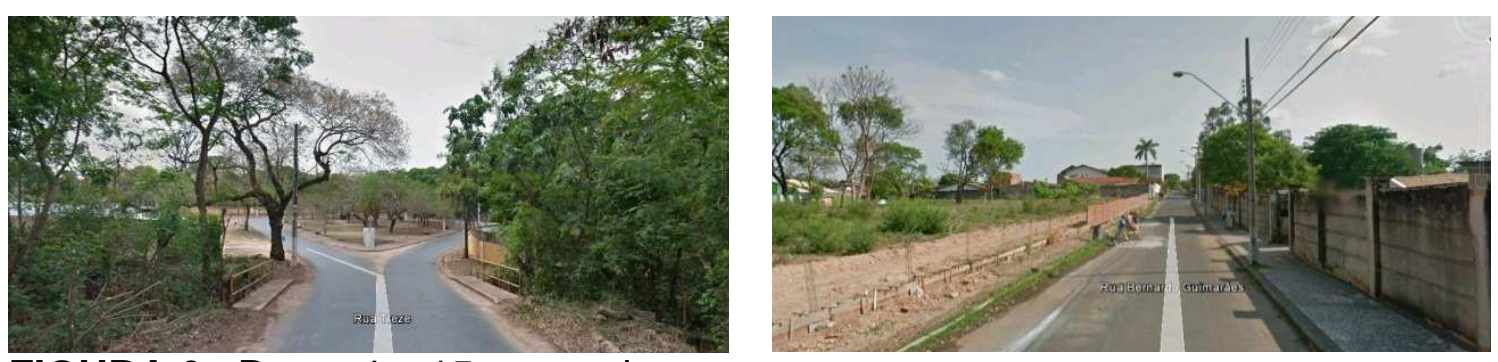

FIGURA 6- Ponto 4 e 15 respectivamente 


\section{Análise da radiação solar dos pontos do transecto}

$\mathrm{Na}$ comparação entre radiação solar e temperatura do ar nota-se que os pontos com altas temperaturas também possuem elevado índice de radiação solar. O ponto com maior radiação solar é o $15 \mathrm{com}$ média de $403 \mathrm{~W} / \mathrm{m}^{2}$ e o menor é o 4 com média de $53 \mathrm{~W} / \mathrm{m}^{2}$ (figura 7 ). A temperatura do ar do ponto 15 é de $32,4 \mathrm{C}$ no período quente-seco e $30,1^{\circ} \mathrm{C}$ no período quente-úmid o e do ponto 4 é de $32,5^{\circ} \mathrm{C}$ no período quente-seco e $29,8^{\circ} \mathrm{C}$ no período quente-úmid o. A diferença de temperatura varia de $0,1^{\circ} \mathrm{C}$ a $0,3^{\circ} \mathrm{C}$.

O ponto 23 de maior temperatura do ar obteve a média de $33,1^{\circ} \mathrm{C}$ no período quente-seco e $30,8^{\circ} \mathrm{C}$ no período quente-úmido. Teve média de radiação solar de $336 \mathrm{~W} / \mathrm{m}^{2}$. O ponto 17 de menor temperatura do ar $\left(29,9^{\circ} \mathrm{C}\right)$ teve média de radiação solar de $112 \mathrm{~W} / \mathrm{m}^{2}$. A diferença entre as médias das temperaturas é de ${ }^{1} \mathrm{C}$. A diferença entre as médias da radiação solar dos pontos 23 e 17 é de $224 \mathrm{~W} / \mathrm{m}^{2}$. Os pontos 4 e 17 estão em locais onde há influência da sombra da vegetação, sendo assim, protegidos da radiação solar.

PAPADAKIS et al. (2001) realizaram um estudo experimental sobre a influência das árvores na transferência de calor de uma fachada orientada a sudoeste e na temperatura interna no ambiente de escritórios, em edifício do Campus da Universidade de Agricultura de Atenas. Instalaram os equipamentos nas áreas sombreadas e expostas da fachada para monitoramento de radiação solar, por um período de quatro dias. As copas das unidades arbóreas bloqueiam a radiação direta pela maior parte do tempo e na área sombreada da fachada só foi registrada radiação difusa. As áreas expostas registram $600 \mathrm{~W} / \mathrm{m}^{2}$ e as sombreadas $100 \mathrm{~W} / \mathrm{m}^{2}$. Os valores só ultrapassaram $100 \mathrm{~W} / \mathrm{m}^{2}$ na parede sombreada, próximo ao meio-dia, quando a altura solar era próxima de $90^{\circ} \mathrm{e}$ a sombra das árvores não era projetada na parede, registrando $180 \mathrm{~W} / \mathrm{m}^{2}$. Já para a energia absorvida pela parede, foi sempre maior na área exposta que na sombreada.

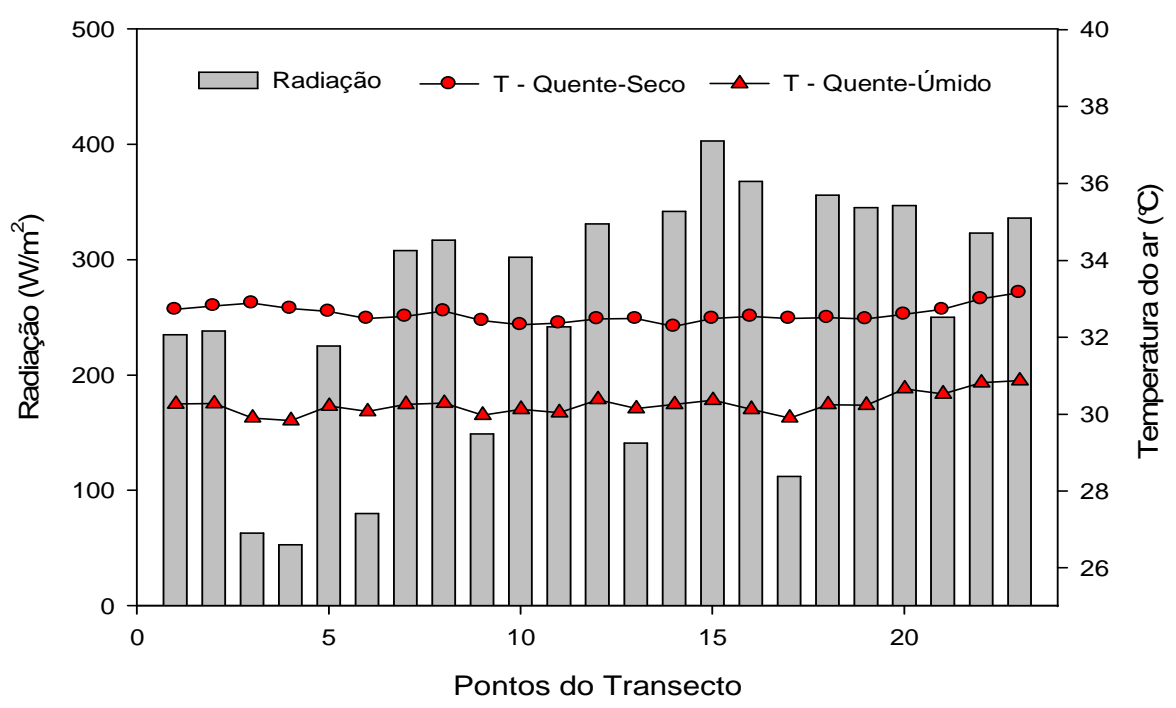

FIGURA 7 - Comparação entre radiação solar e temperatura do ar média 


\section{CONCLUSÕES}

O fator cânion urbano foi considerado representativo no estudo do desempenho microclimático, pois esse fator pode ser responsável pela quantidade de radiação solar incidente na via, e consequentemente o aquecimento da mesma. Como exemplo o ponto 17, com a menor temperatura do ar no período quente-úmido $\left(29,9^{\circ} \mathrm{C}\right)$, é o $3^{\circ} \mathrm{com}$ o maior fator $(\mathrm{H} / \mathrm{W}=1,43)$, enqu anto o ponto $23 \mathrm{com}$ a maior temperatura do ar no período quente-úmido $\left(30,9^{\circ} \mathrm{C}\right)$ tem um fator $(\mathrm{H} / \mathrm{W}=0,13), 11$ vezes menor que o do ponto 17.

A geometria do traçado urbano é responsável por uma considerável contribuição no conforto térmico urbano. Em cidades de clima tropical onde o sombreamento é um fator positivo para melhoria da qualidade ambiental, é necessário considerar-se que as estratégias bioclimáticas que envolvem o formato e tamanho das vias e edificações devem ser pensadas da melhor maneira possível, aliando a isso o sombreamento proporcionado também pelas espécies arbóreas.

A pesquisa apresenta fatores que acarretam mudanças no panorama climático urbano. Sendo assim, o estudo indica algumas questões inerentes ao processo de crescimento da capital mato-grossense, que necessitam ser revistos pelos gestores, a fim de uma melhor tomada de decisão com relação ao conforto ambiental da cidade.

\section{AGRADECIMENTOS}

À FAPEMAT através do Programa de Apoio a Núcleos de Excelência (PRONEX) pelo apoio financeiro ao projeto de pesquisa processo n. 823971/2009. A CAPES e ELETROBRÁS pelo apoio financeiro para esta pesquisa.

\section{REFERÊNCIAS}

ANDREOU, E. The effect of urban layout, street geometry and orientation on shading conditions in urban canyons in the Mediterranean. Renewable Energy, v. 63, p. 587596, 2014.

BARROS, M. P. Dimensão fractal e ilhas de calor urbanas: uma abordagem sistêmica sobre as implicações entre a fragmentação das áreas verdes e o ambiente térmico do espaço urbano. Cuiabá, 2012. 171f. Tese (Doutorado) Programa de Pós-Graduação em Física Ambiental, Universidade Federal de Mato Grosso, 2012.

BOURBIA, F.; BOUCHERIBA, F. Impact of street design on urban microclimate for semi arid climate (Constantine). Renewable Energy, v. 35, p.343-347, 2010.

CUIABÁ. Perfil Socioeconômico de Cuiabá - Volume V Cuiabá - Secretaria Municipal de Desenvolvimento Urbano - SMDU, Setembro, 2012.

EMMANUEL, R.; ROSENLUND, H.; JOHANSSON, E.; Urban shading - a design option for the tropics A study in Colombo, Sri Lanka. International Journal of Climatology, n. 27, p.1995-2004, 2007. 
FRANCO, F. M. Configuração Urbana e sua Interferência no Microclima Local: Estudo de Caso no Bairro do Porto em Cuiabá-MT. 2010. 153f. Dissertação (Mestrado em Física Ambiental), Instituto de Física, Universidade Federal de Mato Grosso, Cuiabá, 2010.

FRANCO, F. M. Análise do Comportamento Termohigrométrico Urbano Sob a Ótica do Uso e Ocupação do Solo em Cuiabá - MT. 2013. 142f. Tese (Doutorado em Física Ambiental), Instituto de Física, Universidade Federal de Mato Grosso, Cuiabá, 2013.

GIVONI B. In: Climate considerations in building and urban design. New York: John Wiley \& sons; 1998.

JOHANSSON, E.; Influence of urban geometry on outdoor thermal comfort in a hot dry climate: A study in Fez, Morocco. Building and Environment, n. 41, p. 13261338, 2006.

KRÜGER, E.; PEARLMUTTER, D.; RASIA, F. Evaluating the impact of canyon geometry and orientation on cooling loads in a high-mass building in a hot dry environment. Applied Energy, n. 87, p. 2068-2078, 2010.

OKE T. R. Street design and urban canopy layer climate. Energy Build, 1988.

PAPADAKIS, G.; TSAMIS, P.; KYRITSTS, S. An experimental investigation of the effects of shading with plants for solar control of buildings. Energy and Buildings, n. 33 p. 833-836, 2001.

PEARLMUTTER, D.; BERLINER, P.; SHAVIV, E. Integrated modeling of pedestrian energy exchange and thermal comfort in urban street canyons. Building and Environment, n. 42, p. 2396-2409, 2007.

PEZZUTO, C. C. Avaliação do ambiente térmico nos espaços urbanos abertos. Estudo de caso em Campinas, SP. 2007. 182 f. Tese (Doutorado) - Faculdade de Engenharia Civil, Arquitetura e Urbanismo da Universidade Estadual de Campinas, Campinas, 2007.

SHASHUA-BAR L.,TSIROS I.; X., HOFFMAN M. E. A modeling study for evaluating passive cooling scenarios in urban streets with trees. Case study: Athens, Greece. Building and Environment, Estados Unidos, v. 45, p 2798-2807, 2010.

STROMANN-ANDERSEN, J., AND P.A. SATTRUP. "The Urban Canyon and Building Energy Use: Urban Density versus Daylight and Passive Solar Gains." Energy and Buildings v. 43, p 2011-2020, 2011. 